\title{
The Physics Potential of a Neutrino Beam from Protvino to KM3NeT/ORCA
}

\author{
Jürgen Brunner, ${ }^{1, *}$ for KM3NeT/P2O \\ ${ }^{1}$ Aix Marseille Univ, CNRS/IN2P3, CPPM, Marseille, France
}

\begin{abstract}
The Protvino accelerator facility located in the Moscow region, Russia, is in a good position to offer a rich experimental research program in the field of neutrino physics. Of particular interest is the possibility to direct a neutrino beam from Protvino towards the KM3NeT/ORCA detector which is currently under construction in the Mediterranean sea $40 \mathrm{~km}$ offshore Toulon, France. Such an experiment, nicknamed P2O (Protvino-to-ORCA), would yield an unparalleled sensitivity to matter effects in the Earth, allowing for the determination of the neutrino mass ordering with a high level of certainty due to its baseline of $2595 \mathrm{~km}$ after only few years of running time at a modest beam intensity up to $100 \mathrm{~kW}$. A second phase of the experiment comprizes a further intensity upgrade of the accelerator complex and a significant densification of the ORCA detector. This would allow for a competitive and complementary measurement of the leptonic CP-violating Dirac phase with a Mton detector but avoiding underground excavation costs.
\end{abstract}

\section{Introduction}

Neutrino physics is one of the most actively developing branches of particle physics, with many fundamental parameters still awaiting to be experimentally determined, as well as great promise for new insights into physics beyond the Standard Model. Some of the key open questions are the presence of charge-parity $(\mathrm{CP})$ violation in the lepton sector, e.g. by the $\mathrm{CP}$-violating Dirac phase in the neutrino mixing matrix and the relative ordering of the three neutrino mass eigenstates ("mass ordering"). These questions can be answered by studying flavour oscillations of $\mathrm{GeV}$ neutrinos over a long baseline ( $\gg 100 \mathrm{~km}$ ). Particle accelerators provide a well controlled environment suited for conducting high precision measurements of that type. Several experiments with different baselines will likely be necessary to cleanly disentangle effects from various poorly constrained parameters, such as the $\mathrm{CP}$ violating phase $\delta_{C P}$, the mass ordering, and (the octant of) the $\theta_{23}$ mixing angle. Furthermore, any new significant experimental finding will need to be independently verified, ideally with an experiment which does not share the same systematic measurement uncertainties. In this regard, the construction of multiple experiments with different baselines is generally well motivated.

With this contribution we would like to create interest in a long-baseline neutrino experiment using the accelerator complex in Protvino (Moscow Oblast, Russia) for generating a neutrino beam and the KM3NeT/ORCA detector in the Mediterranean sea as a far detector.

\footnotetext{
*e-mail: brunner@cppm.in2p3.fr
} 
The scientific potential of the Protvino-to-ORCA (P2O) experiment is presented with an emphasis on the sensitivity to $\mathrm{CP}$ violation $\left(\delta_{C P}\right)$ and neutrino mass ordering. We argue that, thanks to the long baseline $(2595 \mathrm{~km})$ and $8 \mathrm{Mt}$ sensitive volume of the far detector, $\mathrm{P} 2 \mathrm{O}$ would be complementary and competitive to other existing and future long-baseline experiments such as T2K, T2HK, NOvA and DUNE. A vision of the long-term future of $\mathrm{P} 2 \mathrm{O}$ is proposed, including upgrades of the Protvino accelerator complex and the ORCA detector.

\section{$2 \mathrm{KM} 3 \mathrm{NeT} / \mathrm{ORCA}$}

ORCA (Oscillation Research with Cosmics in the Abyss) is one of the two neutrino detectors under construction by the KM3NeT Collaboration [1]. It is located at $42^{\circ} 48^{\prime} \mathrm{N} 06^{\circ} 02^{\prime} \mathrm{E}$, about $40 \mathrm{~km}$ off the coast of Toulon, France, at a depth between $2450 \mathrm{~m}$ (the seabed depth) and $2250 \mathrm{~m}$. When completed, ORCA will consist of 2070 digital optical modules (DOMs) installed on 115 vertical structures (detection units, DUs). With a $9 \mathrm{~m}$ vertical step between the DOMs and a $23 \mathrm{~m}$ horizontal step between the DUs, the total instrumented water volume will approach 8 megatons. ORCA is optimized for the study of atmospheric neutrino oscillations in the energy range between $2 \mathrm{GeV}$ and $30 \mathrm{GeV}$ with the primary goal to determine the neutrino mass ordering. The majority of neutrino events observed by ORCA will be due to electron and muon neutrino and antineutrino charge-current (CC) interactions, with tau neutrinos and neutral current (NC) interactions representing minor backgrounds. ORCA will provide a neutrino energy resolution of $\approx 30 \%$ and a zenith angle resolution of $\approx 7^{\circ}$ at $E_{v}=$ $5 \mathrm{GeV}$. A result with a $3 \sigma$ statistical significance on the mass ordering is expected after three years of data taking [1]. ORCA will also provide improved measurements of the atmospheric neutrino oscillation parameters $\Delta m_{23}^{2}, \theta_{23}$ and constraints on non-standard neutrino interactions, as well as sensitivity to astrophysical neutrino sources, dark matter, and other physics phenomena. The detector construction has recently started and is expected to be completed within $4 \mathrm{yr}$.

\section{The Protvino Accelerator Complex}

The Protvino accelerator complex is located at $54^{\circ} 52^{\prime} \mathrm{N} 37^{\circ} 11^{\prime} \mathrm{E}$, approximately $100 \mathrm{~km}$ South of Moscow, Russia. Its core component is the U-70 synchrotron of $1.5 \mathrm{~km}$ circumference, which accelerates protons up to $70 \mathrm{GeV}$. U-70 was originally built in the 1960 s and has been in regular operation since then. The proton injection chain includes an ion source, a $30 \mathrm{MeV}$ linear accelerator, and a $1.5 \mathrm{GeV}$ booster synchrotron. The accelerator chain is normally operated at a beam energy from $50 \mathrm{GeV}$ to $70 \mathrm{GeV}$, with a proton intensity of up to $1.5 \times 10^{13}$ protons per cycle. The beam cycle is $10 \mathrm{~s}$, with a beam spill duration of up to 3.5 $\mathrm{s}$; or $8 \mathrm{~s}$, with a $5 \mu \mathrm{s}$ beam spill. The complex is operated by the Institute for High Energy Physics (IHEP) which makes part of the "Kurchatov Institute" National Research Center.

The U-70 synchrotron routinely operates at a time-averaged beam power of up to $15 \mathrm{~kW}$. In the 1990s, a new injection scheme was considered at IHEP, which would allow for an increase of the beam intensity to $5 \times 10^{13}$ protons per cycle [2]. Together with the shortening of the cycle to $7 \mathrm{~s}$, this would provide a beam power of $75 \mathrm{~kW}$. In the following, we will use the value of $90 \mathrm{~kW}$ as the achievable goal of such an upgrade. Assuming that the accelerator works for the neutrino program with a $60 \%$ efficiency for 6 months a year, one year of the $90 \mathrm{~kW}$ beam corresponds to $\approx 0.8 \times 10^{20}$ protons on target (POT). Such a beam power is perfectly suited for the ultimate measurement of the neutrino mass ordering at the KM3NeT/ORCA detector. An upgrade up to $450 \mathrm{~kW}$ could be made possible by a new chain of injection accelerators [3]. Note that the design of the main U-70 synchrotron potentially 

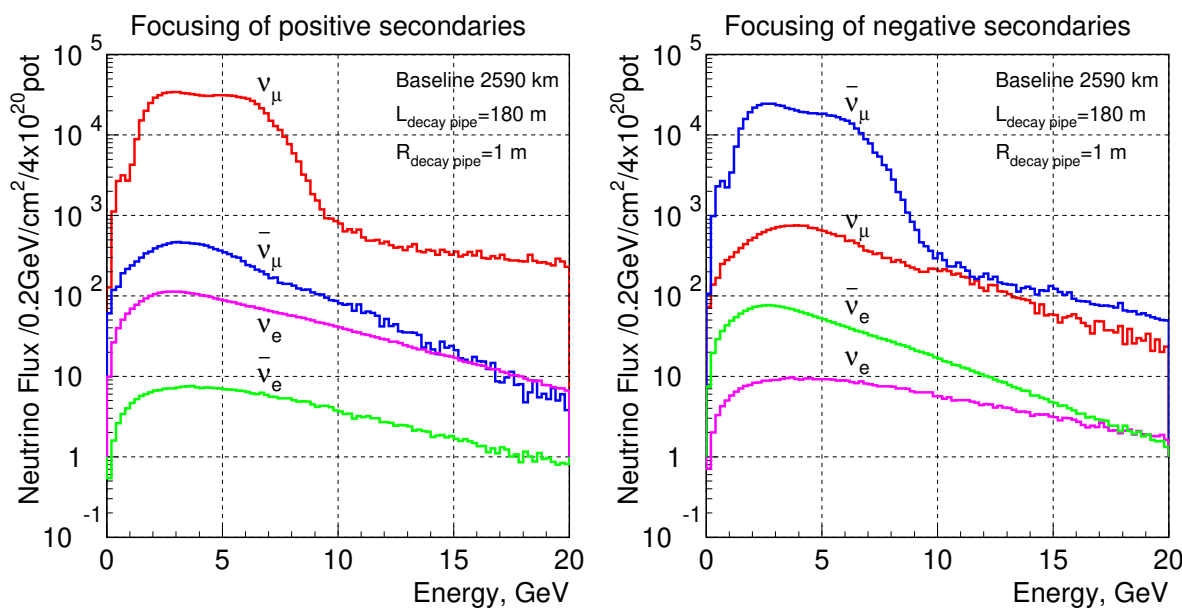

Figure 1. Simulated spectra of the P2O neutrino beam at the ORCA location in neutrino (left panel) and antineutrino (right panel) modes. The absolute normalization is given for $4 \times 10^{20}$ protons on target (POT), which corresponds to $1 \mathrm{yr}$ of operation at the beam power of $450 \mathrm{~kW}$.

allows for operation at a beam power up to $\approx 450 \mathrm{~kW}$. Such a beam power would be adequate for an unequalled measurement $\delta_{C P}$ aiming at a significantly densified version of the ORCA detector.

\section{Neutrino Beamline}

A new neutrino beamline will need to be constructed at Protvino to enable the proposed research program. In order to serve the $\mathrm{P} 2 \mathrm{O}$ long-baseline experiment, the beamline will need to be aligned towards the ORCA site at an inclination angle of $11.8^{\circ}$ (206 mrad) below the horizontal. A baseline design of the neutrino beamline includes the following main components: beam extraction station, which could be installed on an accelerator section located in the main experimental hall; straight section, which delivers the beam from the extraction point to the graphite beam target; secondary beam focusing system using magnetic horns; decay pipe, where neutrinos are produced from pion and kaon decays and beam absorber. The longest section of the beamline is the decay pipe. In the baseline design, the target hall is located at a depth of $\approx 30 \mathrm{~m}$ under ground level, the decay pipe is $\approx 180 \mathrm{~m}$ long (subject to optimization), the absorber hall is $\approx 63 \mathrm{~m}$ below ground level, and the near detector hall is $\approx 90 \mathrm{~m}$ below ground. The magnetic horns will allow for reversal of the electric current polarity in order to choose between the neutrino and antineutrino modes.

A simulation study of the proposed beamline suggests that a $98 \%$ pure muon neutrino beam can be obtained using the $70 \mathrm{GeV}$ proton beam, with a plateau in the neutrino energy distribution between $2 \mathrm{GeV}$ and $7 \mathrm{GeV}$ (see Fig. 1). In the antineutrino mode, a 94\% pure muon antineutrino beam may be obtained [4].

\section{Science with the Neutrino Beam from Protvino to ORCA}

Sending a neutrino beam from Protvino to ORCA would provide a baseline of $2595 \mathrm{~km}$, larger than any accelerator neutrino experiment currently operating or planned elsewhere. 

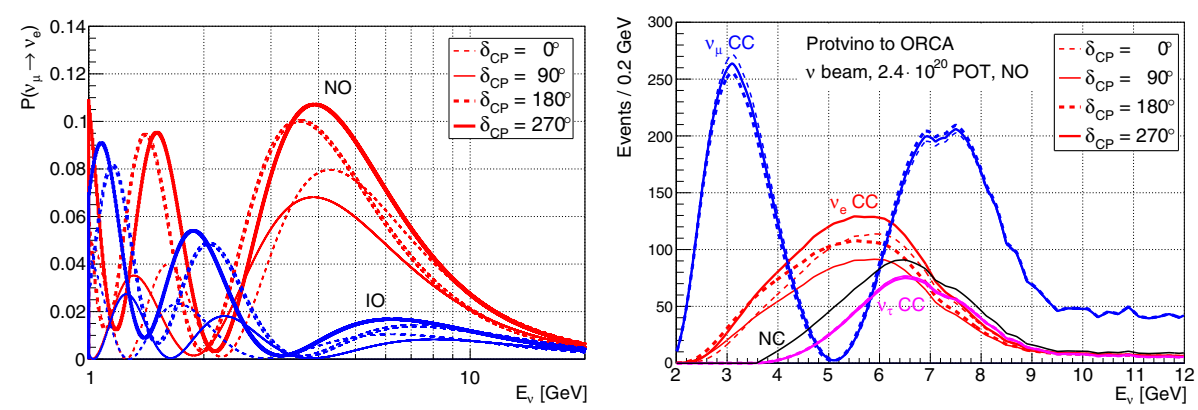

Figure 2. Left : Oscillation probabilities for $v_{\mu} \rightarrow v_{e}$ (electron neutrino appearance) for baseline $\mathrm{L}=$ $2595 \mathrm{~km}$ for normal (red) and inverted (blue) mass ordering. Right: The effect of the CP phase on the expected number of neutrino events that would be detected by ORCA for $2.4 \cdot 10^{20}$ POT. The case of normal neutrino mass ordering with $\theta_{23}=45^{\circ}$ has been assumed. The x-axis shows the true neutrino energy.

The first $v_{\mu} \rightarrow v_{e}$ oscillation maximum would be at $E_{v} \approx 5 \mathrm{GeV}$, within the energy range readily available from the U-70 synchrotron and within the ORCAs nominal energy range. In this energy regime, the neutrino interaction cross section is dominated by deep inelastic scattering, which is relatively well described theoretically (compared to resonant interactions which dominate at $\approx 2-3 \mathrm{GeV}$ ), thus facilitating high-precision measurements of neutrino flavor conversions. For a reference, a recent study by the MINER $v$ A Collaboration reported a $10 \%$ uncertainty for the total neutrino cross section at $2.5 \mathrm{GeV}$ and a $5 \%$ uncertainty at $5 \mathrm{GeV}$ [5]. The $2595 \mathrm{~km}$ baseline is well suited for probing the $\mathrm{CP}$ violating Dirac phase $\delta_{C P}$, as well as for measuring the matter resonance effect $\left(E_{\text {res }}=4 \mathrm{GeV}\right.$ for the Earth crust) [6, 7]. The effects of the mass ordering and $\delta_{C P}$ are most pronounced in the $v_{e}$ appearance channel (see Fig. 2). The large instrument will allow one to detect thousands of neutrino events per year, even with a relatively modest accelerator beam power and despite the very long baseline.

\subsection{Neutrino Mass Ordering and Early Results on CP Violation}

A preliminary study of the scientific potential of the $\mathrm{P} 2 \mathrm{O}$ experiment $[8,9]$ suggests that the neutrino mass ordering could be determined with a 5-10 $\sigma$ statistical significance after five years with a $90 \mathrm{~kW}$ beam based on the striking difference in the $v_{e}$-CC event rate around the first oscillation maximum in the neutrino energy range of 3-6 GeV (see Fig. 3). This would provide a solid confirmation of the $\approx 3 \sigma$ result expected to be obtained in the coming years by ORCA (using atmospheric neutrinos), JUNO (using reactor neutrinos) and NOvA (using accelerator neutrinos).

With an integrated beam intensity of $12 \cdot 10^{20}$ POT, the $\mathrm{P} 2 \mathrm{O}$ experiment could also provide an up to $3 \sigma$ sensitivity to discover $\mathrm{CP}$ violation, assuming a fixed beam polarity chosen to be positive (negative) for the case of normal (inverted) mass ordering. Depending on the true $\delta_{C P}$ value, the accuracy on the value of $\delta_{C P}$ is then $20^{\circ}-40^{\circ}$. The sensitivity estimates quoted here were obtained using a preliminary data analysis pipeline developed for atmospheric neutrino studies [1] and do not include any potential analysis improvements made possible thanks to the known arrival direction and timing of the neutrino beam. The treatment of systematic uncertainties includes a 5\% normalization uncertainty for the combined $v_{\mu}$ and $v_{e}$ event rate, a $10 \%$ uncertainty for $v_{\tau}$, a $5 \%$ uncertainty on the NC event rate, and a $10 \%$ uncertainty on the neutrino flavour identification performance of ORCA. 

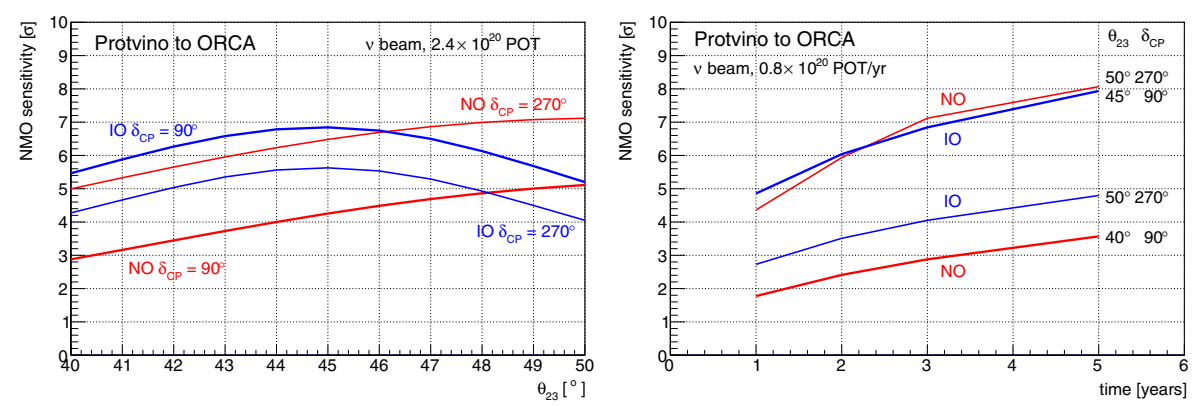

Figure 3. Sensitivity of $\mathrm{P} 2 \mathrm{O}$ to neutrino mass ordering (NMO). Left: NMO sensitivity as a function of the $\theta_{23}$ mixing angle after $3 \mathrm{yr}$ of running with a $90 \mathrm{~kW}$ beam. Right : NMO sensitivity as a function of the accumulated exposure time with the $90 \mathrm{~kW}$ beam. The $\theta_{23}$ and $\delta_{C P}$ values chosen provide the most and the least favorable scenarios for both normal and inverted ordering.

While the sensitivity on $\delta_{C P}$ is encouraging, it is not sufficient in comparison with other future projects. An upgrade on the detector side is needed as well.

\subsection{Future Beyond ORCA}

A more densely-instrumented version of the ORCA detector, called Super-ORCA, is under discussion as a possible next step after ORCA. Super-ORCA could provide a lower energy threshold for neutrino detection, better neutrino flavour identification and better energy resolution compared to ORCA [10]. Such an upgrade could substantially enhance the scientific potential of the $\mathrm{P} 2 \mathrm{O}$ experiment, in particular the accuracy of the $\mathrm{CP}$ phase measurement. A preliminary projection of the sensitivity of Super-ORCA using the Protvino neutrino beam is shown in Fig. 4. In this figure, a 10 times denser detector geometry compared to ORCA is assumed along with a $4 \mathrm{Mt}$ fiducial volume. With such an increased instrumentation density, the energy threshold for neutrino detection is reduced to $\sim 0.5 \mathrm{GeV}$, allowing one to reach the second oscillation maximum, which provides an improved $\delta_{C P}$ sensitivity. The increased instrumentation density also allows one to separate $v_{\mu} / v_{e}$ via the fuzziness of the Cherenkov rings, so that $95 \%$-pure samples of muon-like (dominated by $v_{\mu} \mathrm{CC}$ ) and electronlike events (dominated by $v_{e} \mathrm{CC}$ ) can be selected. The neutrino energy resolution is $\approx 20 \%$ at $E_{v}>1 \mathrm{GeV}$ and is dominated by fluctuations in the number of emitted photons in the hadronic shower [12]. Consequently, such a configuration can yield a 5(9) $\sigma$ sensitivity to discover CP violation after 3(10) years of data taking along with a $7^{\circ}-12^{\circ}$ precision on $\delta_{C P}$ after 10 years, complementary to other long-baseline experiments. It is worth to note that the highest precision is achieved for $\delta_{C P}=\left[90^{\circ}, 270^{\circ}\right]$ complementary to other project which achieve the highest measurement precision for $\delta_{C P}=\left[0^{\circ}, 180^{\circ}\right]$. This is due to the unique capability of the experiment of measuring both the first and the second oscillation maximum in the $v_{e}$ channel as well as CP effects in the $v_{\mu}$ channel in the energy range 2-4 GeV (see Figure 2). Super-ORCA also offers interesting prospects for neutrino oscillation tomography of the Earth and CP violation studies using atmospheric neutrinos [10].

A combined analysis of the atmospheric and accelerator neutrino data collected by ORCA will be possible, improving the systematic uncertainties and parameter degeneracies. Additional science topics with $\mathrm{P} 2 \mathrm{O}$ may include non-standard neutrino interactions and sterile neutrino searches. 

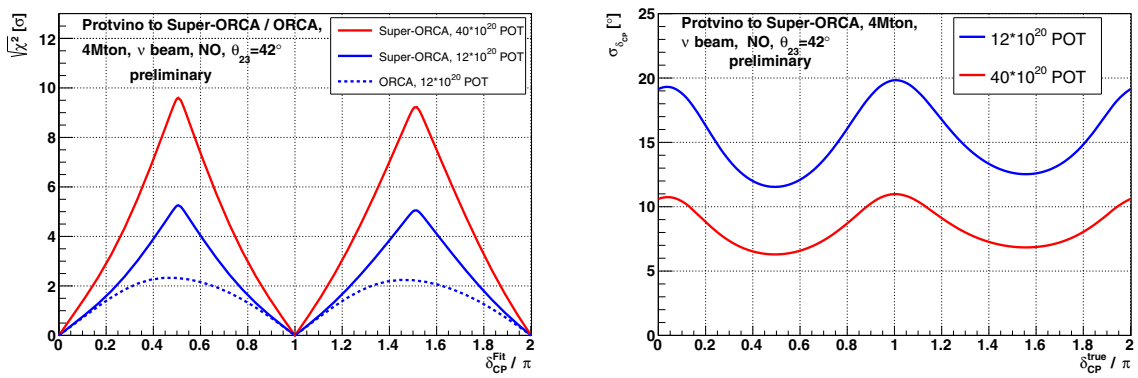

Figure 4. Left: Sensitivities to detect CP violation by operating ORCA 3 years (dashed), Super-ORCA 3 years (solid, blue) and Super-ORCA 10 years (solid-red) in a $450 \mathrm{~kW}$ beam from Protvino. Right : Expected 1- $\sigma$ measurement precision of the CP phase for 3 years (blue) and 10 years (red) data taking with Super-ORCA. (see [10] and [11] for details).

\section{Summary}

The Protvino accelerator facility is well suited for conducting experiments with GeV neutrino beams and has a strong potential to make important contributions to modern neutrino physics. The distance from Protvino to the ORCA neutrino detector in the Mediterranean sea, $2595 \mathrm{~km}$, is ideal for a long-baseline neutrino experiment employing ORCA as far detector. In conjunction with a densification of the ORCA detector, such an experiment promises a competitive and complementary sensitivity to the leptonic CP-violating phase $\delta_{C P}$ while the neutrino mass ordering could already be measured at an early stage of the project.

\section{References}

[1] S. Adrián-Martínez et al. (KM3NeT Collaboration), J. Phys. G 43, 084001 (2016).

[2] IHEP Preprint 93-27 (1993) (in Russian).

[3] N. E. Tyurin et al., News and Problems of Fundamental Physics 2 (2010), http://ihep. ru/files/IHEP-2-10.pdf; http://ihep.ru/files/OMEGA|\%20LOI.pdf.

[4] V. I. Garkusha, F. N. Novoskoltsev, and A. A. Sokolov, IHEP Preprint 2015-5 (2015) (in Russian).

[5] L. Ren et al. (The MINERvA Collaboration), Phys. Rev. D 95, 072009 (2017), Addendum: Phys. Rev. D 97, 019902 (2018).

[6] J. Brunner, Adv. High Energy Phys. 2013, 782538 (2013).

[7] C. Lujan-Peschard, G. Pagliaroli, and F. Vissani, Eur. Phys. J. C73, 2439 (2013).

[8] D. Zaborov (for the KM3NeT Collaboration), Moscow, Russia (2017) arXiv:1803.08017 [hep-ex].

[9] J. Brunner, Heidelberg, Germany (2018) 10.5281/zenodo.1300743.

[10] J. Hofestädt, T. Eberl, and M. Bruchner, Heidelberg, Germany (2018) 10.5281/zenodo.1292936.

[11] M. Bruchner,"Sensitivity Studies for the ORCA and Super-ORCA Neutrino Detector", Master thesis (2018) University of Erlangen-Nuremberg.

[12] S. Adrián-Martínez et al. (KM3NeT Collaboration), JHEP 1705 (2017) 008. 